\title{
Crecimiento postnatal del recién nacido de muy bajo peso (RNMBP). Antropometría a tres años plazo, estudio longitudinal
}

\author{
Mafalda Rizzardini P. ${ }^{1}$; Mario Ferreiro S. ${ }^{2}$; Leticia Felis A. ${ }^{2}$; \\ Lionel Bernier $\mathrm{V}^{2} ;$ M. Angélica Villarroel A. ${ }^{2}$ \\ Three years follow up to postnatal growth in \\ very low birth weight infants
}

\begin{abstract}
Growth of 230 very low birth weight infants (VLBWI) adnitted to the neonatal wards of a metropolitan pediatric hospital at Santiago, Chile was studied prospectively up to 36 months of age, in the period 1980.1988. For further analysis patients were separated in groups A, 60 newborn infants with birth-weight below $1001 \mathrm{~g}$ and $\mathrm{B}$, 170 newborn infants with birth-weight between 1001 and $1500 \mathrm{~g}$. We used Patri's growth charts, to compare the results with fuld term healthy newborns of $3318 \mathrm{~g}$ average birth-weight from the sane socio-economical status. The average weight of group A infants was below 2 SD at age one year and between one and two SD at 2 and 3 years of age. In group $B$ infants weight was between one and 2 SD at one year of age and below 1 SD at 2 years. At age 3 years average weight was very close to normal. Group A infants were not succesfull to achieve the average height of the standard at age 3 year, but this same goal was obtained at 2 years of age in group $B$ infants. Head circumference was within the normal average at ages 3 and 2 vear in group $A$ and $B$ infants, respectivelv.
\end{abstract}

tKey words: verv low birth weight infants, growth, postnatal, height, weight, head circumference.

Actualmente se dispone en Chile de normas nacionales para evaluar el estado nutricional del niño, con las que se han establecido tasas regionales y nacionales de desnutrición. Dichas normas no toman en cuenta el peso de nacimiento, clasificando como desnutridos a muchos lactan. tes y preescolares cuyos pesos pudiesen ser considerados normales en ciertos casos si se aplicasen criterios más apropiados.

Estas consideraciones impulsaron a los auto. res a iniciar en 1989 un estudio longitudinal del crecimiento postnatal de los recién nacidos (RN) de peso inferjor a $1501 \mathrm{~g}$ (RNMBP), cuyos resultados a ocho semanas ${ }^{1}$ y a un año ${ }^{2}$ han sido recientemente comunicados. En esta oportunidad damos a conocer la evolución del peso corporal, talla y perímetro cefálico de una población du. RN de muy bajo peso del área norte de Santiago, desde el nacimiento hasta los tres años de edad. El objetivo principal es proporcionar una curva de crecimiento que, aunque

1. Departamento de Pediatria, Hospital Roberto del Río.

2. Servicio de Pediatr ía, Hospital Roberto del Río. corresponde a una población limitada, puede servir como referencia para otras localidades y para futuras comparaciones en nuestro $y$ otros medios.

\section{Pacientes y Método}

El seguimiento se realizó en 230 RNMBP adecuados para la edad gestacional, libres de anomalías congénitas, displasia broncopulmonar, hemorragia intracraneana, otras enfermedades graves o signos de malnutrición intrauterina. No fueton excluidos los niños que tenían trastornos propios de su inmadurez, aunque hubiesen requerido ventilación mecánica, fototerapia, recambio sanguíneo o alimentación parenteral total. Tados Jos RN fueron atendidos en b unidad de neonatolog ja det hospital Roberto del Río, según sus normas ${ }^{3}$ y dados de alta cuando pesaban, por lo menos, $2000 \mathrm{~g}$. Para el seguimiento fueron examinados una vez al mes en el primer semestre ( \pm 4 días del cumple-mes); cada tres meses el segundo semestre y cada seis meses en los dos últimos afios. Los métodos usados para el control antropométrico fueron los de Patrí y colaboradores, cuyas curvas de crecimiento de niños normales del área norte de Santiago se emplearon con fünes de comparación ${ }^{4}$.

Para el análisjs de los resultados el material se dividió en dos grupos. El grupo A estaba constituido por 60 
recién nacidos con peso al nacer inferior a $1001 \mathrm{~g}$. cuyas características eran: edad gestacional (EG) 27,2 $\pm 1,0$ (variación 24 a 29) semanas; peso al nacer $922,9 \pm$ 79,6 (variación 660 a 1000 ) $g$; talla al nacer (TN) 35,1 × 1.7 (variación 30 a 39 ) $\mathrm{cm}$; perímetro cefálico (PC) $24,5 \pm 1,0$ (variación 22 a 2 8) $\mathrm{cm}$. El grupo B estuwo formado por 170 niños cuyo peso al nacer era de 1001 a $1500 \mathrm{~g}$. EG 29,5 $\pm 1,1$ (varjación 27 a 33) semanas; PN 1233,7 × 71,8 (varjación 1010 a 1500 ) g; TN 38,1. $\pm 1,3$ (variación 34 a 42 ) $\mathrm{cm} ; y$ PC 26,7 $\pm 1,3$ (variación 24 a 30 ) cm. Sesenta y siete niffos $(24,8 \%$ ) abandonaron el control, 12 por fallecimiento antes de los dos años de edad, 8 por bronconeumonía y 4 por causa no precisada.

\section{Resultados}

El incremento ponderal máximo en los njños del grupo $A$ ocurió entre el cuarto y quinto mes de vida y alcanzó a $826 \mathrm{~g}$. En los del grupo B a $931,8 \mathrm{~g}$, entre el tercero y cuarto mes. Al año de edad los primeros pesaban $6859,8 \mathrm{~g}$, a los dos años $9670,4 \mathrm{~g}$ y a los tres afios $11923,4 \mathrm{~g}$. A estas mismas edades, los del grupo B pesaban en promedio $7784,1-10700,1 \mathrm{y}$ $13103,1 \mathrm{~g}$, respectivamente (tablas 1 y 2 ).

El incremento máximo de la talla ocurió entre el segundo y tercer mes en los niños del grupo A y entre el tercero y cuarto en el grupo B $(4,1$ y $4 \mathrm{~cm}$, respectivamente). Al año los nifios del grupo A median en promedio $66,4 \mathrm{~cm}$, a los dos años $79,1 \mathrm{~cm}$ y a los tres años $90,2 \mathrm{~cm}$.

Tabla 1

Peso (promedio \pm 1 desviación estándar) según edad post ratal e incrementos mensuales en recién nacidos de peso de nacimiento $\measuredangle$ a $1000 \mathrm{~g}$

\begin{tabular}{llcll}
$\begin{array}{l}\text { Edad } \\
\text { meses }\end{array}$ & $\begin{array}{c}\text { Peso } \bar{x} \\
\mathrm{~g}\end{array}$ & $1 \mathrm{DE}$ & Incremento mensual \\
\hline
\end{tabular}

\begin{tabular}{rrrrr}
\hline 0 & 60 & 922,9 & 79,6 & - \\
1 & 60 & 1178,7 & 102,2 & 255,8 \\
2 & 60 & 1577,3 & 326,8 & 398,6 \\
3 & 59 & 2273,4 & 537,2 & 696,1 \\
4 & 58 & 2969,8 & 553,9 & 696,4 \\
5 & 56 & 3795,1 & 614,7 & 826,0 \\
6 & 55 & 4370,8 & 667,7 & 575,7 \\
9 & 50 & 5750,9 & 702,2 & 460,0 \\
12 & 50 & 6855,8 & 760,5 & 368,3 \\
18 & 46 & 8272,4 & 835,5 & 236,1 \\
24 & 46 & 9670,4 & 911,4 & 233,0 \\
30 & 46 & 10894,4 & 1048,3 & 204,0 \\
36 & 45 & 11923,4 & $1,152,1$ & 171,5 \\
\hline
\end{tabular}

DE: Desviación estándar.
Tabla 2

Peso (promedio \pm 1 desviación estándar) según edad postnatal e irucrementos mensuales en recién nacidos de peso de nacimiento entre 1001 y $1500 \mathrm{~g}$

\begin{tabular}{|c|c|c|c|c|}
\hline $\begin{array}{l}\text { Edad } \\
\text { meses }\end{array}$ & n & $\begin{array}{c}\text { Peso } \bar{x} \\
g\end{array}$ & $1 \mathrm{DE}$ & Incremento mensual \\
\hline 0 & 170 & 1233,7 & 71,8 & - \\
\hline 1 & 170 & 1379,5 & 217,0 & 145,8 \\
\hline 2 & 170 & 2055,5 & 385,2 & 676.0 \\
\hline 3 & 168 & 2941,4 & 567,6 & 885,9 \\
\hline 4 & 160 & 3873,2 & 583,0 & 931,8 \\
\hline 5 & 152 & 4588,1 & 706,2 & 714,9 \\
\hline 6 & 150 & 5333,8 & 766,6 & 745,7 \\
\hline 9 & 142 & 6800,2 & 802,0 & 488,8 \\
\hline 12 & 145 & 7784,1 & 960,7 & 328,0 \\
\hline 18 & 140 & 9324,9 & 992,3 & 256,8 \\
\hline 24 & 130 & 10700,1 & 1177,1 & 229,2 \\
\hline 30 & 132 & 11903,1 & 1224,7 & 200,5 \\
\hline 36 & 128 & 13103,1 & 1429,5 & 200,0 \\
\hline
\end{tabular}

DE: Desviación estándar.

Los del grupo B: $71,2-86,2$ y $91,4 \mathrm{~cm}$, respectivamente (tablas 3 y 4 ).

El perímetro cefálico experimentó también un rápido aumento en los primeros meses. En el gnupo a el incremento máximo ocurrió entre el segundo $y$ tercer mes $(3,3 \mathrm{~cm})$ y en el grupo $B$ entre el primero y el segundo $(3,7 \mathrm{~cm})$. Al año de edad el perímetro cefálico de los RN del grupo A medía en promedio $43,1 \mathrm{~cm}$, a los dos

Tabla 3

Talla (promedio \pm 1 desviación estándar) según edad postnatal e incrementos mensuales en recién nacidos de peso de nacimiento inferior a $1001 \mathrm{~g}$

\begin{tabular}{|c|c|c|c|c|}
\hline $\begin{array}{l}\text { Edad } \\
\text { meses }\end{array}$ & n & $\begin{array}{c}\text { Talla } \bar{x} \\
\mathrm{~cm}\end{array}$ & $1 \mathrm{DE}$ & Incremento mensual \\
\hline$\theta$ & 60 & 35,1 & 1,7 & - \\
\hline I & 60 & 37,0 & 2,0 & 1,9 \\
\hline 2 & 60 & 40,1 & 2,1 & 3,1 \\
\hline 3 & 59 & 44,2 & 2,1 & 4,1 \\
\hline 4 & $5 ?$ & 47,8 & 2,3 & 3,6 \\
\hline 5 & 56 & 51,4 & 2,5 & 3,6 \\
\hline 6 & 55 & 54,6 & 2,8 & 3,2 \\
\hline 9 & 49 & 61,7 & 3,0 & 2,5 \\
\hline 12 & 50 & 66,4 & 3,2 & 2,5 \\
\hline 18 & 46 & 73,2 & 3.7 & 1,1 \\
\hline 24 & 45 & 79,1 & 4,0 & 1,0 \\
\hline 30 & 46 & 84,6 & 4,0 & 0,9 \\
\hline 36 & 45 & 90,2 & 4,2 & 0,9 \\
\hline
\end{tabular}

DE: Desviación estándar. 
Tabla 4

Talla (promedio \pm 1 desviación estándar) segín edad postnatal e incrementos mensuales en recién nacidos de peso de nacimiento entre 1001 y $1500 \mathrm{~g}$

$\begin{array}{llccc}\begin{array}{l}\text { Edad } \\ \text { meses }\end{array} & \text { h } & \begin{array}{c}\text { Talla } \bar{x} \\ \text { cm }\end{array} & 1 \mathrm{DE} & \text { Incremento mensual }\end{array}$

\begin{tabular}{rrrrr}
\hline 0 & 170 & 38,1 & 1,3 & - \\
1 & 170 & 40,7 & 2,0 & 2,6 \\
2 & 170 & 43,8 & 2,1 & 3,1 \\
3 & 168 & 47,6 & 2,3 & 3,8 \\
4 & 160 & 51,6 & 2,3 & $\mathbf{4 , 0}$ \\
5 & 152 & 55,1 & 2,4 & 3,5 \\
6 & 150 & 58,3 & 2,6 & 3,2 \\
9 & 142 & 65,2 & 2,6 & 2,3 \\
12 & 145 & 71,2 & 2,7 & 2,0 \\
18 & 140 & 79,6 & 3,0 & 1,4 \\
24 & 130 & 86,2 & 3,0 & 1,1 \\
30 & 122 & 89,3 & 3,1 & 0,5 \\
36 & 128 & 91,4 & 3,2 & 0,4
\end{tabular}

DE: Desviación estándar.

años $46,5 \mathrm{~cm}$ y a los tres años $48,2 \mathrm{~cm}$. El de los niños del grupo B: $44,4 \cdot 47,3$ y $49 \mathrm{~cm}$, respectivamente (tablas 5 y 6 ).

En las figuras 1 y 2 se grafican estos resultados en curvas levemente suavizadas. A pesar que aún en los niños más pequeños existe un rápido crecimiento inicial, el peso de los $\mathrm{RN}$ de menos de $1001 \mathrm{~g}$, al año y dos años de edad, está bajo 2 DE del promedio considerado como normal,

Tabla 5

Perimetro cefálico ( \pm l desviación estándar) según edad postnatal e incrementos mensuales en recién nacidos de peso de naciniento inferior a $1001 \mathrm{~g}$

$\begin{aligned} & \text { Edad } \\ & \text { meses }\end{aligned}$
$\begin{gathered}\text { Perímetro } \\ \text { cmálico } \vec{x} \\ \text { cm }\end{gathered}$

\begin{tabular}{rllll}
\hline 0 & 60 & 24,5 & 1,0 & $\overrightarrow{0}$ \\
$\mathbf{1}$ & 60 & 26,5 & 1,0 & 2,0 \\
2 & 60 & 29,6 & 1,2 & 3,1 \\
3 & 58 & 32,9 & 1,2 & 3,3 \\
4 & 57 & 35,1 & 1,2 & 2,2 \\
5 & 56 & 36,8 & 1,3 & 1,7 \\
6 & 54 & 38,1 & 1,4 & 1,3 \\
9 & 50 & $\mathbf{4 1 , 4}$ & 1,4 & 1,1 \\
12 & $\mathbf{5 0}$ & $\mathbf{4 3 , 1}$ & 1,5 & 0,6 \\
18 & $\mathbf{4 5}$ & 45,5 & 1,6 & 0,4 \\
24 & $\mathbf{4 6}$ & $\mathbf{4 6 , 5}$ & 1,6 & 0,2 \\
30 & $\mathbf{4 5}$ & 47,4 & 1,6 & 0,2 \\
36 & $\mathbf{4 5}$ & $\mathbf{4 8 , 2}$ & 1,7 & 0,1 \\
\hline
\end{tabular}

DE: Desviación estándar.

\section{Tabla 6}

Perímetro cefálico $( \pm 1$ desviación estándar) según edad postnatal e incrementos mensuales en recién racidos de peso de nacimiento entre 1001 y $1500 \mathrm{~g}$

\begin{tabular}{ccccc}
\hline $\begin{array}{c}\text { Edad } \\
\text { meses }\end{array}$ & $\begin{array}{c}\text { n } \\
\text { Per imetro } \\
\text { cefálico } \bar{x} \\
\mathrm{~cm}\end{array}$ & $\mathbf{1 ~ D E}$ & Incremento mensual \\
\hline & & & & \\
0 & $\mathbf{1 7 0}$ & 26,7 & 1,3 & - \\
1 & $\mathbf{1 7 0}$ & 28,9 & 1,4 & 2,2 \\
2 & $\mathbf{1 7 0}$ & 32,6 & 1,4 & 3,7 \\
3 & 166 & 34,9 & 1,5 & 2,3 \\
4 & 160 & 37,0 & 1,5 & 2,1 \\
5 & 152 & 38,6 & 1,5 & 1,6 \\
6 & 150 & 40,0 & 1,6 & 1,4 \\
$\mathbf{9}$ & $\mathbf{1 4 0}$ & 42,7 & 1,6 & 0,9 \\
12 & 145 & 44,4 & 1,7 & 0,6 \\
18 & 140 & 45,9 & 1,9 & 0,3 \\
24 & 130 & 47,3 & 1,9 & 0,2 \\
30 & 130 & 48,2 & 2,0 & 0,2 \\
36 & 128 & 49,0 & 2,2 & 0,1 \\
\hline
\end{tabular}

DE: Desviación estándar.

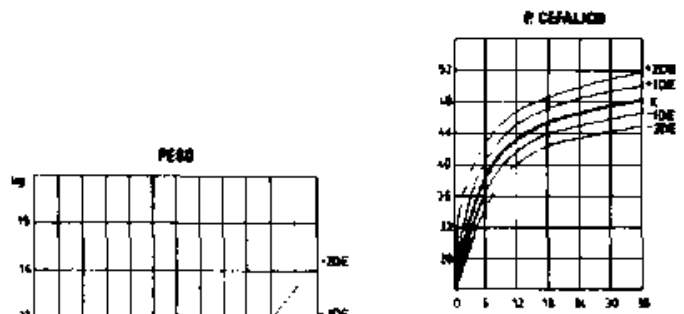

Thila

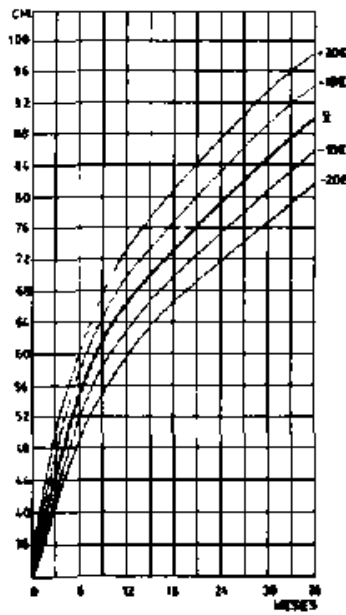

Figura 1: Peso, talla y perímetro cefálico según edad postnatal en RN de peso inferior a $1001 \mathrm{~g}$ (promedio \pm 1 y 2 desviación estándar). 

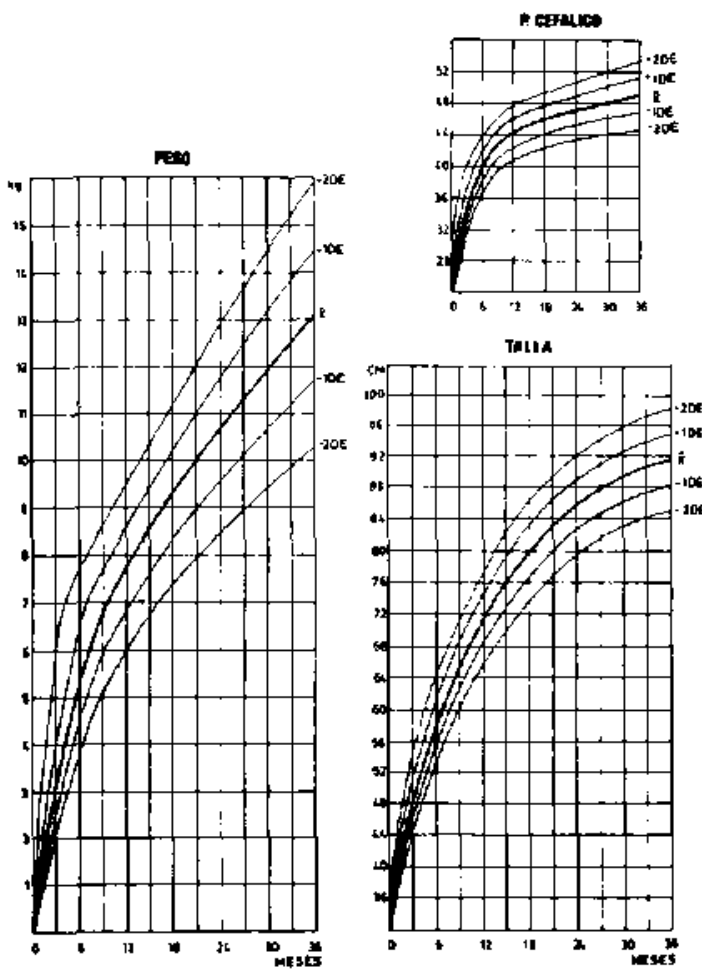

Figura 2: Peso, talia y perímetro cefálico según edad postnatal en RN de 1001 a $1500 \mathrm{~g}$ (promedio \pm 1 y 2 desuiación estánđar).

bajo 1 DE a los 3 anoos. La talla sigue un curso paralelo al peso y pennanece bajo $2 \mathrm{DE}$ hasta los 24 meses. El perímetro cefálico, aunque tiene un crecimiento más rápido, no alcanza el promedio normal a los tres años de vida extrauterina. Los niños de 1001 a $1500 \mathrm{~g}$ crecen más rápidamente que los priłneros: a los 12 meses el peso está bajo $2 \mathrm{DE}$ del promedio normal, a los 24 meses bajo 1 DE y a los 3 años muy cerca de él. Tanto la talla como el perímetro cefálico se normalizan a los 24 meses.

\section{Comentario}

Se ha postulado que el crecimiento ideal del RNMBP es aquel que reproduce el del feto in utero y que, corregida la edad, no debieran haber diferencias con los RN a término. Desafortunadamente el crecimiento postnatal inicial es más lento que el prenatal y si a esto se agrega el descenso de peso de los primeros días, fácil es comprender que las metas ideales son prácticamente inalcanzables.
Exister dificultades para construir una curva "normal" de crecimiento RNMBP por lo reducido del material disponíble y la gran cantidad de eventos que ocurren en las primeras semanas de vida, los que, de alguna manera, influyen en el estado nutritivo. La literatura nacional, aunque escasa $y$ con casuísticas limitadas ${ }^{5}$ ha proporcionado hasta ahora valiosa información sobre lo que podría considerarse como crecimiento normal del RNMBP procedente de un medio socioeconómico poco favorecido. Los resultados obtenidos no se diferencian sustancial. mente de los de ésta y otras experiencias en poblaciones preferentemente caucásicas. Al año de edad el RN de 1001 a $1500 \mathrm{~g}$ no sobrepasa $\operatorname{los} 8 \mathrm{~kg}$, a los dos años los $11 \mathrm{~kg}$ y a los tres afos los $13 \mathrm{~kg}$, con una diferencia cercana a 1 $\mathrm{kg}$ a los tres aflos, para los de peso inferior a $1001 \mathrm{~g}$. El ideal seria poder contar con antropometría de RNMBP procedentes de comunidades más pudientes, pero en estos casos, además de la situación socioeconómica, factores genéticos podrían, según algunos ${ }^{6}$, distorsionar los resultados, de modo que lo estimado como normal para los niños con uno o ningún progenitor de origen nativo pudiera no serlo para el que tiene dos o tres antepasados de este origen. Si se analizan los resultados de Cerezo? en Guatemala, pareciera que la talla materna tiene gran influencia sobre el peso del RNMBP en el primero y segundo año de vida. Esta relación entre talla, perímetro cefálico de la madre y antropometría postnatal del RNMBP ha sido observada también por otros 8 .

Aunque la población estudiada en nuestro y otros medios no es estrictamente igual, la mayor parte de los autores coinciden en que la curva de erecimiento del RNMBP, después de las primeras semanas de vida, sigue un curso paralelo al de los nacidos a término pero en niveles más bajos y que los niños cuyo peso de nacimiento es inferior a $1001 \mathrm{~g}$, son más livianos durante los primeros tres años de vida, aunque la talla y eI perímetro cefálico se acercan más al promedio nomal ${ }^{9-12}$. En estudios longitudinales futuros habria que determinar si este menor peso se mantjene durante más tiempo.

\section{Resumen}

Se describe en forma prospectiva el crecimiento postnatal, hasta los 36 meses de edad, de 230 
RNMBP atendidos en el hospital Roberto del Río de Santiago đe Chile entre los años 1980 y 1988. Para el análisis de los resultados se dividieron los pacientes en dos grupos: en el grupo A fueron incluidos 60 RN con peso inferior a $1001 \mathrm{~g}$, y en el grupo B $170 \mathrm{RN}$ cuyo peso al nacer variaba entre 1001 y $1500 \mathrm{~g}$. Para comparar los resultados se usan las curvas de crecimiento de niños sanos con peso de nacimiento promedio de 3318 g de Patrí y colaboradores, pertenecientes al mismo medio socioeconómico. El peso de los niffos del grupo A está bajo 2 DE del promedio normal al año de edad y entre I y 2 DE a los 2 y 3 ax̃os. El peso de los niños del grupo B estaba entre 1 y 2 DE al año, bajo $1 \mathrm{DE}$ a los dos âfos y muy cerca del promedio nomal a los 3 años. La talla no llegó al promedio normal del estándar empleado a los 3 afios, en los niffos del grupo A, pero esta meta fue alcanzada a los 2 años en los del grupo B. El perímetro cefálico estaba dentro del promedio normal a los 3 y 2 años, en los grupos A y B, respectivamente.

(Palabras clave: recién nacidos de muy bajo peso al nacer, crecimiento postnatal, peso, talla, perimetro craneano.)

\section{Referencias}

1. Rizzardint $M$, Ferretro $M$, Nilo P, Palominos $M A$, Pontoja $S$ : Crecimiento postnatal del RN de muy bajo peso I: Crecimiento inmediato. Rev Chil Pediatr 1988; 59: 166-169.
2. Rizzardini $M$, Ferreiro $M$, Berwier $I$, Bernier P. Crecimiento postnatal del recién nacido de muy bajo peso I]: Antropometría a un año plazo, eśtudio longitudinal. Rev Chil Pediatr 1989; 60: 5-10.

3. Aguiló C, Emparonza E, Rizzardini M: Normas de atención pediátrica (tercera edición). SantiagoChile: Publicaciones Técnicas Mediterráneo, 1989.

4. Patri $A$, Sepulveda $H$, Vatenzuela $C$, Cortés $O$ : Antropometria del niño chileno de 0 a 6 años. Santiago-Chile: Editorial Andrés Bello, 1984.

5. Weldt $E$, Hering $E$, Valenzuela B, Angulo $Y$. Neira A: Seguimiento de niños con peso de nacimiento inferior a $1500 \mathrm{~g}$ Rev Chil Pediatr 1986; 57: 51-56.

6. Rona $R$, Pierret $T$ : Genotipo en niñas adolescentes de Santiago. Rev Međ Chile 1973; 101: 207211.

7. Cerezo R, Fipueroa $R$, Venoncio $J$, Howard $f$ : Pronóstico, crecimiento y desarrollo del niño de muy bajo peso al nacer en relación al sexo. Guate mala Pediátrica 1988; 10: 216-222.

8. Ross $G$, Lipper $E$, Auld $P$ : Physical growth and development outcome in very low-birth-weight premature infants at 3 years of age. $J$ Pediatr 1985; 107 : 284-286.

9. Hack $M$, Merkatz I, MeGrath $S$ et al. Catch-up growth in very-low-birth-weight infants. AJDC $1984 ; 138: 370 \cdot 375$.

10. Ross G. Kratuss A, Auld P: Growth achievement in low-birth-weight premature infants. Relationship to neurobehavioral outcome at one year. I Pediatr 1983; 103: 105-108.

11. Gill A. Yu V, Bajuk B, Astbury J: Postnatal growth in infants born before 30 weeks gestation. Arch Dis Child 1986; 61: 549-553.

12. Kimble $K$, Ariagnon $R$, Stevenson $D$, Sunshine $P h$ : Growth to age 3 years among very low birthweight sequelae-free survivors of modern neonatal intensive care. J Pediatr 1982; 100: 622-624.

Esta publicación está disponible en copias de microfilms de 16 y $35 \mathrm{~mm}$ y microfichas de $105 \mathrm{~mm}$, las que pueden solicitarse a:

University Microfilms International

300 North Zeeb Road

Ann Arbor, Michigan 48106, USA.

This journal is also available in $16 \mathrm{~mm}$ microfilm, $35 \mathrm{~mm}$ microfílm and $105 \mathrm{~mm}$ microfilm copies through University Microfilms International, 300 North Zeeb Road, Ann Arbor, Michigan 48106, USA. 